\title{
The Effect of Inflammation on the Healing Process of Acute Skin Wounds Under the Treatment of Wounds with Injections in Rats
}

This article was published in the following Dove Press journal: Journal of Experimental Pharmacology

\author{
Victor Stupin (D' \\ Natalia Manturova $\mathbb{D}^{2}$ \\ Ekaterina Silina $\mathbb{1}^{3}$ \\ Petr Litvitskiy ${ }^{4}$ \\ Vitaly Vasin' \\ Elena Artyushkova ${ }^{5}$ \\ Alexander Ivanov ${ }^{6}$ \\ Mikhail Gladchenko ${ }^{5}$ \\ Salekh Aliev (D) \\ 'Department of Hospital Surgery No I, \\ Pirogov Russian National Research \\ Medical University, Moscow, Russia; \\ ${ }^{2}$ Department of Plastic and \\ Reconstructive Surgery, Cosmetology \\ and Cell Technologies, Pirogov Russian \\ National Research Medical University, \\ Moscow, Russia; ${ }^{3}$ Department of Human \\ Pathology, I.M. Sechenov First Moscow \\ State Medical University (Sechenov \\ University), Moscow, Russia; \\ ${ }^{4}$ Department of Pathophysiology, I.M. \\ Sechenov First Moscow State Medical \\ University (Sechenov University), \\ Moscow, Russia; ${ }^{5}$ Research Institute of \\ Experimental Medicine, Kursk State \\ Medical University, Kursk, Russia; \\ ${ }^{6}$ Department of Histology, Embryology, \\ and Cytology, Kursk State Medical \\ University, Kursk, Russia
}

Purpose: To study the effects of inflammation on the healing process of rats' acute skin wounds during treatment with different injections.

Methods: The study was carried out on Wistar rats, on which square wounds were simulated in the back region. Four groups of wounds were studied. On the day of the simulation (day 0 ), solutions of the drugs were injected into the wounds: an isotonic sodium chloride solution (Control group), mesenchymal stem cells (SC group), collagen (Collagen group), and a deproteinized hemoderivative of calf blood (DHB group). Within 2 weeks, the wound healing process was assessed by observing and calculating changes in the wound areas, temperatures, and epithelialization levels. On days 3, 7, and 14, wound tissue samples were taken for histological examination, morphological analysis of the healing process, and quantitative assessment of granulation layers' leukocyte infiltration.

Results: A correlation between the process of inflammation and epithelization during the healing of skin wounds was established. The anti-inflammatory effect of SC injection on the wound edge tissues was determined, as well as the pro-inflammatory effect of DHB, and the absence of effects on the inflammation course under the collagen treatment. Compared to the control group, the transition from the exudative phase of inflammation to the proliferative phase was faster, as well was wound epithelialization in the SC and Collagen groups. A negative correlation between the level of tissue temperature in the center of wounds and their area were recorded, which intensified over time.

Conclusion: The severity and duration of the inflammation process during wound healing were ambiguous with the use of different injection treatments. This should compel clinicians to use different markers of drug therapy effectiveness during wound healing. Excessive leukocyte infiltration with a low temperature of wounds and a large scab were markers of delayed wound healing.

Keywords: skin wound, inflammation, leukocytes, epithelialization, collagen, stem cells, deproteinized hemoderivative of calf blood

\section{Introduction}

The acute skin wound healing process goes through several successive stages. ${ }^{1-5}$ The first stage is hemostasis. The duration of this stage does not exceed several hours after injury. The second stage of the healing process is inflammation, whose duration varies between 3 and 7 days. In this case, the short alteration phase during inflammation is replaced by the exudation phase and then by the proliferation phase. The proliferation phase usually lasts up to 3-4 weeks after injury and ends
Correspondence: Ekaterina Silina Department of Human Pathology, I.M. Sechenov First Moscow State Medical University (Sechenov University),

Trubetskaya Str, 8, Moscow II999I, Russia

Email silinaekaterina@mail.ru
Journal of Experimental Pharmacology 2020:12 409-422

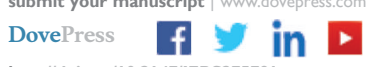

(c) (1) (5) 2020 Stupin et al. This work is published and licensed by Dove Medical Press Limited. The full terms of this license are available at https://www.dovepress.com/terms. cc) work you hereby accept the Terms. Non-commercial uses of the work are permitted without any further permission from Dove Medical Press Limited, provided the work is properly attributed. For permission for commercial use of this work, please see paragraphs 4.2 and 5 of our Terms (https://www.dovepress.com/terms.php). 
(normally) with complete epithelization of the wound surface. The last stage is wound remodeling; this process lasts up to one year and relates not to wound healing but the formation and transformation of cicatricial deformities. Thus, one of the key stages of wound healing is the inflammation phase, which is a transition process between wound hemostasis and proliferation - that is, the period from the moment of skin damage to the restoration of the barrier and other functions of the skin., ${ }^{1,3-5}$

Inflammation is an evolutionarily developed process that facilitates an organism's defense against damage to its tissues and organs. The process of wound inflammation is crucial for the optimal completion of hemostasis, as well as the detection and elimination of pathogenic microorganisms, the removal of damaged tissues, and wound cleaning. $^{2,5-7}$ These steps progress via the participation of leukocytes, which migrate from the bloodstream to the lesion site. The migration is followed by the formation and release of cytokines, reactive oxygen species, and phagocytosis. $^{6-10}$ As the elements of innate immunity, leukocytes are the first tools of organism defense and perform the functions of the epidermis and dermis that have not yet formed. In addition, leukocytes (mainly macrophages) are responsible for the timely and adequate preparation of wound tissues for proliferation. ${ }^{11-15}$ It is known that during an optimal process of wound healing and the absence of infection, neutrophils, which prevail in the wound for 2-5 days after injury and actively migrate into the wound along the chemokine gradient up to 3 days after injury, are eliminated from the wound after the completion of the phagocytosis cycle. To replace active neutrophil infiltration of the wound 3-5 days after injury, monocytes (mononuclear cells) are recruited into the wound, which then differentiate into macrophages, which are important participants in the transition from the inflammation phase to the proliferative phase during the healing process. ${ }^{13-17}$

However, if the acute inflammation is inadequate and prolonged (lasting 7 or more days), the inflammation becomes pathogenic and is associated with impaired interactions between different types of cells (including fibroblasts and keratinocytes, which ensure the formation of new layers of skin). ${ }^{1,4,18}$ This can lead to a chronic nonhealing wound with bacterial colonization, repeated episodes of ischemia-reperfusion injury, and the development of cellular and systemic distress reactions. ${ }^{12,18,19}$ Inadequate hyper inflammation leads to the formation of excessive and pathological scar tissue at the end of the wound remodeling process. ${ }^{20-22}$ This has functional, cosmetic, psychological, and economic implications. The annual market for chronic wound dressings exceeds \$25 billion and $\$ 12$ billion for cicatricial deformities. ${ }^{23}$ Thus, the most important stage of the wound healing process is the inflammation's intensity and duration, as well as the transition of its exudative phase to the proliferative one.

Currently, numerous pharmacological agents can inhibit the inflammation process. Classic examples are the anti-inflammatory drugs used for rheumatic or autoinflammatory disease treatment. There are also drugs for wound treatment that can affect and inhibit the transition from the exudative to the proliferation phase, either directly or indirectly. In this regard, it is important to evaluate the benefits and drawbacks of various drugs that can change the inflammatory response and possibly accelerate the transition from exudation to proliferation.

To resolve this issue, we organized an experimental study to identify the effect of inflammation on the healing process of an acute skin wound under the direct injection of agents that stimulate healing.

To achieve this goal, acute skin wounds were modeled via small prototype operations performed under local anesthesia in surgery and cosmetology, which outnumber all other types of surgical interventions. As pharmacological agents, injectable forms with different mechanisms of action were chosen. It is known that mesenchymal stem cells have an anti-inflammatory effect. ${ }^{24-28}$ Deproteinized Calf Blood Medicine (Actovegin ${ }^{\mathbb{B}}$ preparation) is used in everyday clinical practice as it significantly affects regional blood flow and the intensity of local inflammation. ${ }^{29-33}$ Collagen I type $\left(\mathrm{Collost}^{\circledR}\right)$ does not significantly affect inflammation but is effective as a wound healing stimulator. $^{34-39}$

\section{Materials and Methods}

The experimental study was performed on Wistar rats of the same sex (males) and age (9 months) weighing $436.7 \pm$ 5.5 grams.

\section{Ethical Considerations}

The experiment was performed under the principles of laboratory animal handling and complies with the provision of the "European Convention for the Protection of Vertebral Animals Used for Experimental and Other Scientific Purposes". The study was approved at the meeting of the Regional Ethics Committee of the Kursk State Medical University under the Ministry of Health of the 
Russian Federation (Protocol No. 5 dated 02.11.17). The SCs used in the experiment were isolated from a human umbilical cord after a normal birth after 38-40 weeks of gestation with the informed consent of the donors.

\section{Research Design and Groups}

Before the experiment, the rats were kept in quarantine for 2 weeks under standard vivarium conditions. Subsequently, the animals were randomized by weight and labeled. Next, the skin wounds were modeled (day 0 ) on the backs. Following this, the dynamics of wound healing were studied for 14 days. During the entire period of the experiment, the animals were kept in individual cages with free access to food and water and a 12/12 light regime.

Wounds were modeled under non-sterile conditions using general anesthesia (chloral hydrate $300 \mathrm{mg} / \mathrm{kg}$ intraperitoneally). On the shaved skin of the back, at the same distance on both sides of the spine, square wounds of the same size (the length of each side was $11 \mathrm{~mm}$, with the depth of wounds extending to the fascia) were formed using a special device for creating standardized wounds (Patent Rus No. 79701/10.01.2009). Ten minutes after modelling, the wound area averaged $127.7 \pm 2.0 \mathrm{~mm}^{2}$.

Before the experiment, the animals were divided into four groups with different treatments in each. Initially, there were 28 animals in each group, but before modeling, the animals were marked so that 7 wounds were eliminated at each control point.

On day $0,0.2 \mathrm{~mL}$ of various solutions were injected into the inferolateral angles of all wounds in all animal groups (isotonic saline solution of $0.9 \%$ sodium chloride (Control group); culture of mesenchymal stem cells isolated from a human umbilical cord, 100,000 cells per wound (stem cell group, SC); collagen in the form of Collost 7\% (Collagen group); deproteinized hemoderivative of the calf blood in the form of the Actovegin drug, 40 $\mathrm{mg} / \mathrm{mL}$ (DHB group)).

\section{Research Control Points}

On day 0 of the study, the wounds were modeled, the baseline parameters (wound size and wounds center temperature) were recorded, and the drugs or saline solution were injected depending on the randomization group.

The dynamic assessment of the indicators; the examination, measurement, and photographing of the wounds; and temperature measurements were carried out on days 1 , 3,7 , and 14 . On days 3,7 , and 14 , the animals were removed from the experiment for labeling. Euthanasia was performed under general anesthesia (chloral hydrate $300 \mathrm{mg} / \mathrm{kg}$ intraperitoneally) by rapid exsanguination of the rat (dissecting the right ventricle of the heart). After euthanasia, histological probes of the wound edge tissues were prepared.

\section{Research Methods}

To assess the dynamics of the wound healing process, the surface sizes of the wounds and the temperatures of the wound bottoms and edges were studied. Light microscopy of histological probes from the wound bottoms and edges was also performed to count the number of leukocytes in the wounds per unit area.

This paper presents an analysis of the rate of change on the wound surface area, which was calculated by the formula (Sx - S0)/S0 x 100\%, where S0 is the wound area on the modeling day, and Sx is the wound area at the control points on days $1,3,7$, and 14 . The final result is expressed as a percentage of the original size. The wound area, limited by its edges, was calculated using the JMicroVision 1.2.7 software (Switzerland) based on photographs of the wounds taken at checkpoints via a unified technique (Canon EOS550D camera, Japan, Jpeg format). The wound tissue temperature was measured in the center from the same distance using a Fluke VT02 infrared thermometer (Fluke, USA).

Wound tissues were sliced sequentially for histological analysis. The slices were stained with Hematoxylin-eosin (for descriptive light microscopy) or only hematoxylin (for processing in the Image-J program, National Institute of Health, USA, with a quantitative count of the leukocyte number per $\mathrm{mm}^{2}$ ). During the analysis, the slices were divided into three sectors (the central tissues and two opposite edge tissues). Each area equaled $33.3 \%$ of the total transverse size of the wound. Descriptive microscopy at $\mathrm{x} 40, \mathrm{x} 100$, and $\mathrm{x} 400$ magnification was performed using Levenhuk D740 (USA) and the Leica CME (Germany) microscopes.

Research data were statistically analyzed using the SPSS 23.0 software (IBM Company, USA) according to standard parametric and nonparametric criteria. Descriptive statistics of the continuous quantitative data are presented as the mean and standard error of the mean, as well as the median (Me) and the values of the lower (25\%) and upper (75\%) quartiles. The Mann-Whitney $U$ test was used to compare two independent nonparametric samples, and the Wilcoxon test was used for two 
dependent samples. Differences were considered to be significant at $p<0.05$. The correlation analysis was performed using the Pearson and Spearman methods.

\section{Results}

\section{Changes in the Wound Area}

Analysis of the proportional change in the wound area on the 1st day (24 hours after modeling the wounds) demonstrated an increase of the wound area in $100 \%$ of the Control and DHB group animals. On average, in these groups, the area increased by $31 \%$ and $25 \%$, respectively, compared to the initial areas of the same wounds on the day of modeling $(\mathrm{p}<0.05)$. In the SC and Collagen groups, no statistically significant dynamics of wound area increase were observed. At the same time, the SC group wounds decreased in size by $5 \%$, on average, in 24 hours (in $57 \%$ of the animals, the areas of the wounds increased, while the wounds decreased in $43 \% ; p>0.05$ ). The wound areas in the Collagen group rats did not change on day 1 compared to day 0 (the area decreased in half of the cases and increased in the other half; the median was $0.7 \%$; $>$ $0.05)$. There were also statistically significant differences: After 24 hours, in the SC and Collagen groups, the wound areas were less than those in the Control and DHB groups.

By the end of the 3rd day of the study, the Control group's wound areas were, on average, $26 \%$ larger than those on day $0(\mathrm{p}<0.05)$, and the DHB group was $11 \%$ larger $(\mathrm{p}<0.05)$. An increase in the wound area was recorded in $89 \%$ of cases in the Control group and in $82 \%$ of cases in the DHB group. The wound area did not statistically change compared to day 0 in the Collagen group $(\mathrm{Me}=0.9 \% ; \mathrm{p}>0.05)$. A statistically significant reduction in the wound area was recorded only in the SC group: On day 3, the reduction was, on average, $9 \%$ (p $<0.05$ ), and a reduction in size was noted in $89 \%$ of the cases. The wound areas of the Control and DHB groups were significantly larger than those in the SC and Collagen groups. A statistically significant decrease in wound area compared to the Control by the end of the 3rd day was observed in the SC group, with a smaller decrease in the Collagen group. The wound areas of the DHB and Control groups were similar and decreased less than those in the Collagen and SC groups.

By the end of the 7th day, the wound surface area decreased in all groups, but the degree of wound healing varied. The wound areas in the Collagen group decreased to the greatest extent $(\mathrm{Me}=-39 \%)$, while the control wounds demonstrated the smallest decrease $(\mathrm{Me}=-2 \%)$. The wound areas of the SC and DHB groups, on average, decreased by $26 \%$ and $28 \%$, respectively. However, several cases of a wound area increase were recorded. These cases were registered only in the Control (38\%) and DHB (24\%) groups. All SC and Collagen group wounds decreased by the end of the 7th day. Thus, the SC and Collagen groups were better than the Control group, while DHB had the least effect on wound healing. Once injected into the wound tissue, the stem cells gradually lost their effectiveness by the end of the week. This was due to both the limited lifespan of stem cells in the wound and the presence of histone incompatibility markers on the surfaces of the cells injected. As a result, the proportional changes in the wound areas of the SC and DHB groups were the same by this time. Collagen injected into the wound tissue still potentiated healing at the end of the 7th day. Despite the absence of statistically significant differences between the SC and Collagen rats in terms of the wound area change dynamics, in the Collagen group, the changes were 19.5 times more intense than those in the control wounds $(p<0.05)$ and 1.4 times more intense than those in the DHB group $(p<0.05)$.

At the end of the 14th day, all wound areas decreased: the areas in the Control group decreased by $80 \%$ on average, those in the DHB group by $86 \%$, those in the SC group by $88 \%$, and those in the Collagen group by $91 \%$. Thus, the various injectable drugs (DHB, SC, and Collagen) that were used to treat the wounds accelerated the wound healing process by day 14 compared to control $(p<0.05)$. At the same time, the introduction of SC or Collagen solutions into the wound edges on the day of modeling ultimately provided prominent results that were statistically significantly different from the use of DHB (Table 1).

\section{Wound Center Temperature}

The wound center tissue temperatures of the Control group averaged $35.3{ }^{\circ} \mathrm{C}$ by the end of the $1^{\text {st }}$ day and did not statistically differ from the Collagen and DHB groups ( $p>$ 0.05 ), even though the wound temperature was highest in the Collagen group (median $=35.45{ }^{\circ} \mathrm{C}$ ). The lowest temperature of the wound center on day 1 was observed in the SC group $\left(33.6{ }^{\circ} \mathrm{C}\right.$, which was $1.4-1.9^{\circ} \mathrm{C}$ less than that in the other groups, $\mathrm{p}<0.05$ ).

Thus, 24 hours after the wound modeling, an increase in size was recorded in the Control and DHB groups, which directly correlated with an increase in the wound 
Table I Changes in the Wound Area Compared to Their Initial Size on Day 0 (\%) in the Different Treatment Groups

\begin{tabular}{|l|l|l|l|l|}
\hline Groups & Day I & Day 3 & Day 7 & Day I4 \\
\hline Control & $31.0[17.1: 41.0]$ & $25.6[5.3: 35.8]$ & $-1.9[-15.2: 11.5]$ & $-79.9[-83.6:-71.3]$ \\
Stem cells & $-5.0[-14.1: 10.5]^{*}$ & $-9.0[-24.9:-4.4]^{*} \#$ & $-26.3[-37.4:-12.6]^{*} \#$ & $-88.4[-92.9:-86.1]^{*} \#$ \\
Collagen & $0.7[-8.8: 10.9]^{*}$ & $-0.9[-8.1:-13.6]^{*}$ & $-39.1[-45.7:-25.0]^{*} \#$ & $-91.5[-95.2:-88.2]^{*} \#$ \\
Deproteinized hemoderivative of calf blood & $25.2[-18.1: 45.3]$ & $11.2[1.8: 24.5]$ & $-28.4[-37.2:-5.3]$ & $-85.7[-89.7:-83.0]^{*} \#$ \\
P & SC/DHG & SC/Collagen & Collagen/DHG & SC/DHG \\
& Collagen/DHG & SC/DHG & & Collagen/DHG \\
& & Collagen/DHG & & \\
\hline
\end{tabular}

Notes: Median [lower $25 \%$ quartile: upper $75 \%$ quartile]. \# - the difference in the associated indicator dynamics within the same group compared to day 0 (Wilcoxon test, $p<0.05$ ). *-statistically significant difference from the control group (Mann-Whitney test, $\mathrm{p}<0.05$ ). P- significant difference between two unrelated treatment groups (Mann-Whitney test, $\mathrm{p}<0.05$ ): Collagen/DHG, Collagen and Deproteinized hemoderivative of calf blood; SC/Collagen, Stem cells and Collagen; SC/DHG, Stem cells and Deproteinized hemoderivative of calf blood.

center temperature and indicated a higher intensity of the inflammatory process. The lowest wound center temperature and the best dynamics of wound area reduction were recorded in the SC group, whose wounds decreased by $5 \%$ on average.

The maximum wound center temperature by the end of the 3rd day was the highest in the $\mathrm{SC}$ group $\left(\mathrm{Me}=35.6^{\circ} \mathrm{C}\right)$, where it was significantly higher than that in the Control, DHB, and Collagen groups by $1.2^{\circ} \mathrm{C}(\mathrm{p}<0.05), 0.8^{\circ} \mathrm{C}(\mathrm{p}<0.05)$, and 0.6 ${ }^{\circ} \mathrm{C}(\mathrm{p}<0.05)$, on average, respectively. There were also statistically significant differences in the wound tissue temperature in the $\mathrm{SC}$ and Collagen $\left(0.6^{\circ} \mathrm{C}\right.$ more in the $\mathrm{SC}$ group, $\mathrm{p}$ $<0.05)$, SC and DHB $\left(1.1^{\circ} \mathrm{C}\right.$ more in the SC group, $\mathrm{p}<0$, $05)$, and Collagen and DHB groups $\left(0.5{ }^{\circ} \mathrm{C}\right.$ higher in the Collagen group $(\mathrm{p}<0.05)$.

The wound tissue temperature on the 7 th day of the study was lower than that on the $3 \mathrm{rd}$ day by an average of $0.6^{\circ} \mathrm{C}$ in all groups. This may be due to the transition from the exudative inflammation phase to the proliferation phase. A comparative analysis showed that the highest wound center temperatures were located in the $\mathrm{SC}\left(\mathrm{Me}=35.0^{\circ} \mathrm{C}\right)$ and Collagen $\left(\mathrm{Me}=34.7^{\circ} \mathrm{C}\right)$ groups, which also demonstrated the highest rates of wound healing. The wound center temperature was lower in the DHB group $\left(\mathrm{Me}=34.1^{\circ} \mathrm{C}\right)$, with the lowest in the Control $\left(\mathrm{Me}=33.8^{\circ} \mathrm{C}\right)$ group. The groups with high (SC and Collagen) and low wound center temperatures (Control and DHB) statistically significantly differed in this indicator on the 7 th day.

The wound center temperature on the 14th day after modeling decreased compared to the 7 th day in the Control and Collagen groups but increased in the $\mathrm{DHB}$ and $\mathrm{SC}$ groups. The temperature of the wound tissue on day 14 was, on average, the lowest in the Control $\left(\mathrm{Me}=33.2{ }^{\circ} \mathrm{C}\right)$ and $\mathrm{DHB}\left(\mathrm{Me}=34.3^{\circ} \mathrm{C}\right)$ groups. Due to the high variability of the results, there were no significant differences among the indicators in these groups $(p>0.05)$. The maximum tissue temperature in the wound center was recorded in the $\mathrm{SC}$ group $\left(\mathrm{Me}=35.9{ }^{\circ} \mathrm{C}\right)$, which was, on average, $1.6{ }^{\circ} \mathrm{C}$ higher than that in the DHB group $(\mathrm{p}<0.05), 1.8^{\circ} \mathrm{C}$ higher than that in the Collagen group $(\mathrm{p}<0.05)$, and $2.7^{\circ} \mathrm{C}$ higher than that in the Control group $(\mathrm{p}<0.05))$. The wound temperature in the Collagen group averaged $34.1^{\circ} \mathrm{C}$, which did not differ from the DHB group but was, on average, $0.9{ }^{\circ} \mathrm{C}$ higher than that in the Control group $(\mathrm{p}<0.05)$ (Table 2$)$. The

Table 2 Wound Center Temperature $\left({ }^{\circ} \mathrm{C}\right)$ in the Different Treatment Groups

\begin{tabular}{|l|l|l|l|l|}
\hline Groups & Day I & Day 3 & Day 7 & Day I4 \\
\hline Control & $35.3[34.4: 35.6]$ & $34.4[33.8: 34.9]$ & $33.8[33.0: 34.7]$ & $33.2[32.0: 34.4]$ \\
Stem cells & $33.6[32.4: 34.7]^{*}$ & $35.6[35.1: 36.1]^{*}$ & $35.0[34.5: 35.6]^{*}$ & $35.9[35.4: 36.3]^{*}$ \\
Collagen & $35.5[34.9: 35.9]$ & $35.0[34.7: 35.4]^{*}$ & $34.7[34.4: 35.4]^{*}$ & $34.1[33.4: 35.0]^{*}$ \\
Deproteinized hemoderivative of calf blood & $35.0[34.5: 35.6]$ & $34.5[34.3: 35.0]$ & $34.1[33.5: 34.5]$ & $34.3[31.9: 34.7]$ \\
P & SC/Collagen & SC/Collagen & SC/DHG & SC/Collagen \\
& SC/DHG & SC/DHG & Collagen/DHG & SC/DHG \\
& & Collagen/DHG & & \\
\hline
\end{tabular}

Notes: Median [lower 25\% quartile: upper 75\% quartile]. *- statistically significant difference from the control group (Mann-Whitney test, $\mathrm{P}<0.05$ ). P- significant difference between two unrelated treatment groups (Mann-Whitney test, $\mathrm{p}<0.05$ ): Collagen/DHG, Collagen and Deproteinized hemoderivative of calf blood; SC/Collagen, Stem cells and Collagen; SC/DHG, Stem cells and Deproteinized hemoderivative of calf blood. 
high variability in tissue temperatures might be explained by significant differences in the structures and thicknesses of the scabs (clearly visible upon histological examination).

The latter argument may be confirmed by the persistent negative correlation of the increasing dynamics between the tissue temperature levels in the wound centers and their areas. Thus, the wound temperatures determined on the 1 st day of the study were negatively correlated with the wounds on day $7(\mathrm{r}=-0.175 ; \mathrm{p}<0.01)$ and $14(\mathrm{r}=-0.147$; $\mathrm{p}<0.01)$. The wound tissue temperature on day 3 was negatively correlated with the wound areas on day $3(\mathrm{r}=$ $-0.108 ; \mathrm{p}<0.05)$, day $7(\mathrm{r}=-0.204 ; \mathrm{p}<0.01)$, and day 14 $(\mathrm{r}=-0.298 ; \mathrm{p}<0.01)$. The wound tissue temperature on day 7 was negatively correlated with the wound areas on day $7(\mathrm{r}=-0.201 ; \mathrm{p}<0.01)$ and day $14(\mathrm{r}=-0.199 ; \mathrm{p}$ $<0.01)$. Thus, in terms of dynamics, the steadily decreasing wound temperature during the first week of the healing process was associated with a slowdown in healing and the large wound areas on days 3, 7, and 14 of the study.

\section{Morphometric and Histological \\ Parameters}

Wound State by the End of the 3rd Day of the Study Morphometrically, all wound tissues by the end of the 3rd day showed signs of exudative inflammation, including dilation and a plethora of blood vessels, swelling of the tissues up to the subcutaneous fat, the emigration of leukocytes from the bloodstream, and granulation infiltration by leukocytes.

The exudative inflammation phase proceeded differently in the various groups, as demonstrated by the wound center and edge leukocyte data analysis in the different groups by the end of day 3 of the study (Table 3 ).

Table 3 The Number of Leukocytes in the Center and Edges of Wounds On Day 3

\begin{tabular}{|l|l|l|}
\hline Groups & Center & Edges \\
\hline Control & $210[206: 217]$ & $176[163: 181]$ \\
Stem cells & $180[167: 182] *$ & $130[102: 139] *$ \\
Collagen & 201 [19I: 2I7] & I2I [107: 137]* \\
Deproteinized & $203[195: 226]$ & $114[$ [108: 145]* \\
hemoderivative of calf blood & & \\
P & SC/Collagen & - \\
& SC/GDB & \\
\hline
\end{tabular}

Notes: Median [lower $25 \%$ quartile: upper $75 \%$ quartile].*- statistically significant difference from the control group (Mann-Whitney test, $\mathrm{p}<0.05$ ). $\mathrm{P}-$ significant difference between two unrelated treatment groups (Mann-Whitney test, $\mathrm{p}<0.05$ ): SC/Collagen, Stem cells and Collagen; SC/DHG, Stem cells and Deproteinized hemoderivative of calf blood.
An increase in wound size on day 3 was morphologically combined with an increase in the leukocyte numbers in the wound centers and edges. By the end of the 3rd day, the maximum number of leukocytes in the center and edge was recorded in the Control group ( $\left(\mathrm{Me}=210\right.$ cells per $\left.1 \mathrm{~mm}^{2}\right)$ and $\left(\mathrm{Me}=176\right.$ leukocytes $\left./ \mathrm{mm}^{2}\right)$, respectively). The leukocyte numbers in the wound edges of the control group were, on average, 1.35 times higher than those in the SC group (p $<0.05$ ), 1.54 times higher than those in the DHB group ( $p$ $<0.05$ ), and 1.45 times higher than those in the Collagen group $(\mathrm{p}<0.05)$. In the SC group, the wound area was the smallest on day 3. The number of leukocytes in the bottom tissue was also the smallest $\left(\mathrm{Me}=180\right.$ leukocytes $/ \mathrm{mm}^{2}$ ) and significantly lower than that in all other groups $(\mathrm{p}<0.05)$.

Direct weak significant correlations were established between the wound area and the total number of leukocytes on day 3 in both the wound centers $(r=0.321 ; p$ $<0.05)$ and their edges $(r=0.286 ; p<0.05)$. Consequently, the maximum levels of leukocyte infiltration of the wound edges and bottom tissues by day 3 can serve as a marker of persistent acute inflammation and might be the cause of a possible slowdown in the process of wound repair, as well as the formation of a coarser postoperative scar in subsequent time periods. The preservation of a larger area of wounds in the DHB and Control groups, where there were also more leukocytes, confirms this assumption.

The histological wound biopsy analysis on the 3rd day showed a scab in the center of all wounds located on a thin layer of granulation tissue covering the bottom of the wound. At the same time, deep sections of the scab were infiltrated with polymorphonuclear leukocytes. In all wound tissues, signs of the venous plethora of the hypodermis, the fascial vascular plexus, and the phenomenon of the "marginal pool of leukocytes" (accumulation of leukocytes in the parietal blood layer of the postcapillary and collecting venules) were found. The number of leukocytes in the parietal layer of blood vessels indicated their ongoing migration into the paravasal tissue, which is typical for the exudative inflammation phase.

All wound surfaces of the Control group were covered by scabs, which were the thickest and densest among the other groups. Leukocytes then infiltrated and spread widely into the granulation tissue and the deeper layers of the dermis (Figure 1).

The largest scab infiltration by polymorphonuclear leukocytes was observed In the DHB group alongside significant wound tissue swelling up to the subcutaneous fatty tissue, in which dilated and full-blooded vessels were found everywhere (Figure 2). 
In the Collagen group, by the end of the 3rd day, the scabs were also located on the underlying granulation tissue. However, in contrast to the control group of animals, leukocyte infiltration was less severe here, despite the significant thickness of the scabs. As in the control group, the underlying tissue was edematous, with signs of venous congestion. In some wounds the Collagen group, a formed layer of horizontally located fibroblasts moderately infiltrated by mononuclear cells was visualized in the granulation tissue immediately adjacent to the wound bottom (Figure 3 ).
In the SC group wounds, the scab severity (in terms of width and density), as well as the degree of leukocyte infiltration, was the smallest compared to the other group wounds. At the same time, tissue edema and granulation in the wound bottoms were strongly marked. Compared to the other wound groups, macrophages (monocytes) and lymphocytes predominated over neutrophils in the wound infiltration of the SC group (Figure 4).

Rarely, in rats of the $\mathrm{SC}(\mathrm{n}=1)$ and Collagen $(\mathrm{n}=2)$ groups, a newly formed thin epidermis was visualized in the
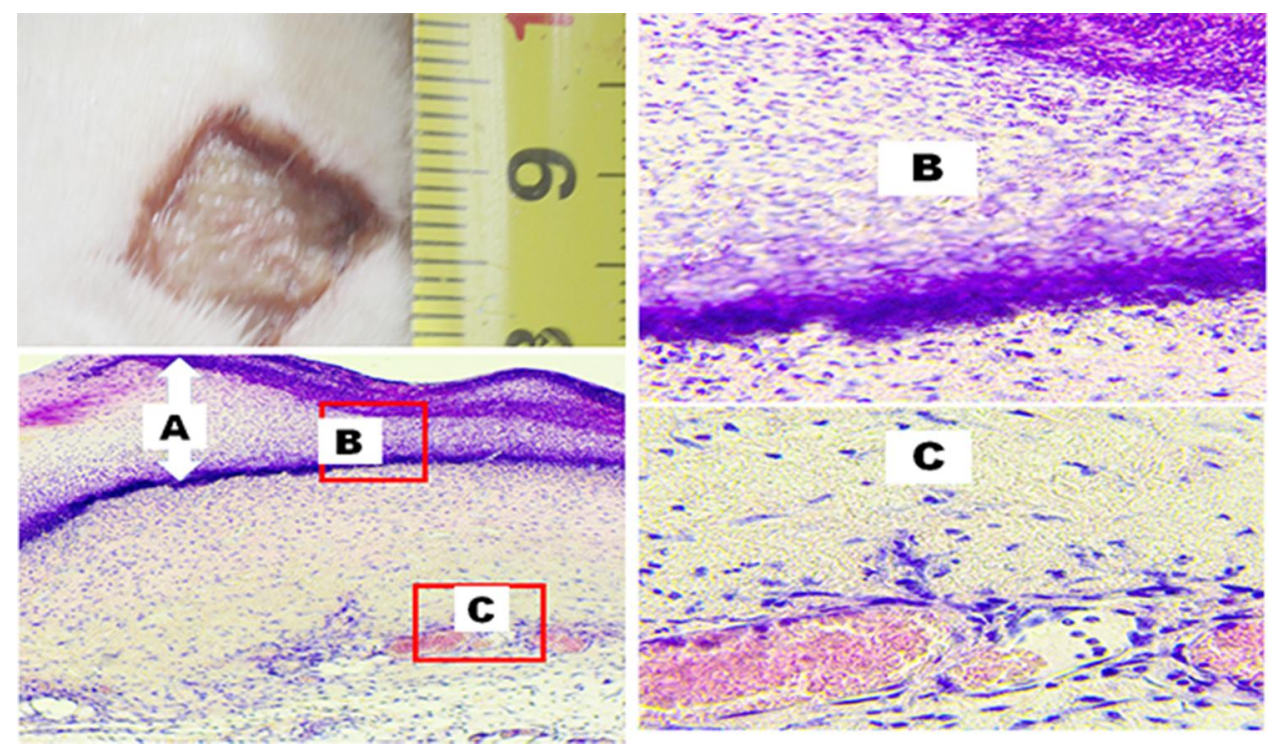

Figure I The wound center by the end of the 3rd day after wound modeling, Control group. Hematoxylin-eosin staining. Magnification: $X 100$ (bottom left) and $\times 400$ (right). Large scab (A), dense leukocyte infiltrate at the granulation tissue borders and in the scab (B), magnification $\times 400)$, leukocytes in scab deep layers, where the congested vessels are dilated $((\mathbf{C})$, magnification $\times 400)$.

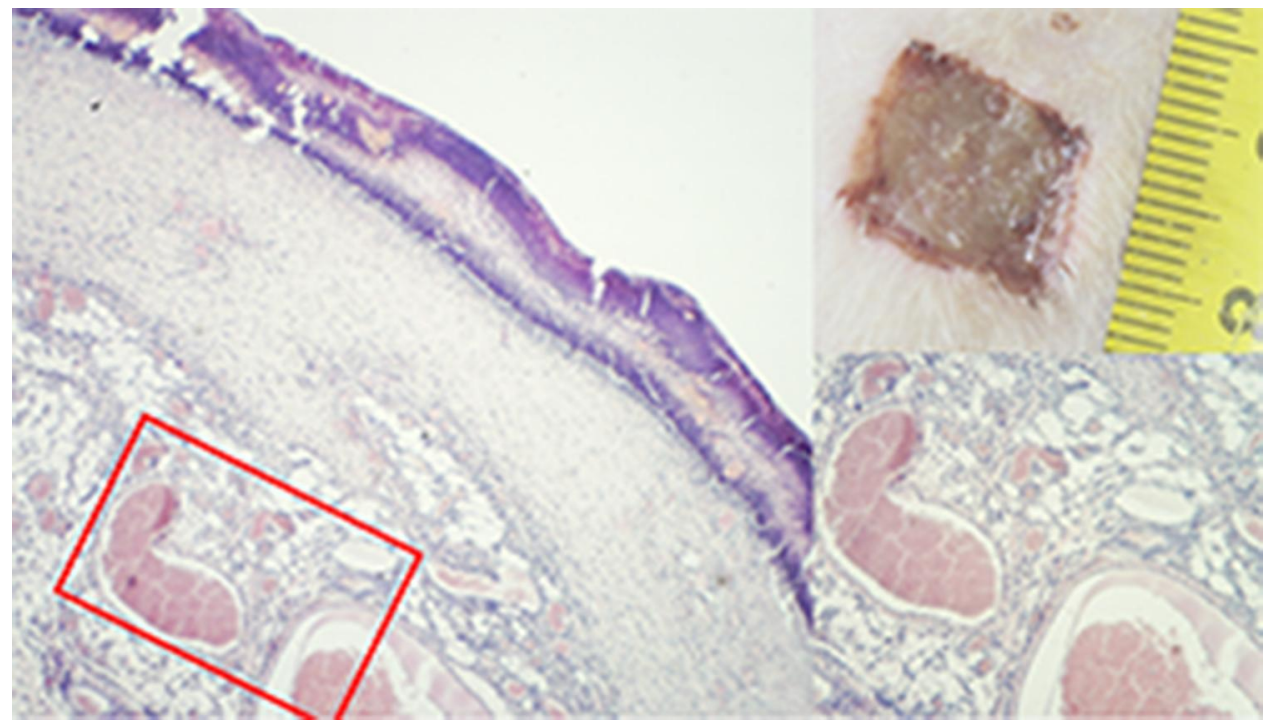

Figure 2 The wound crater center by the end of the 3rd day, DHB group. Abundant scab infiltration, tissue edema, and vessel plethora. Hematoxylin-eosin staining (Magnification: $\times 40$ and $\times 100)$. 


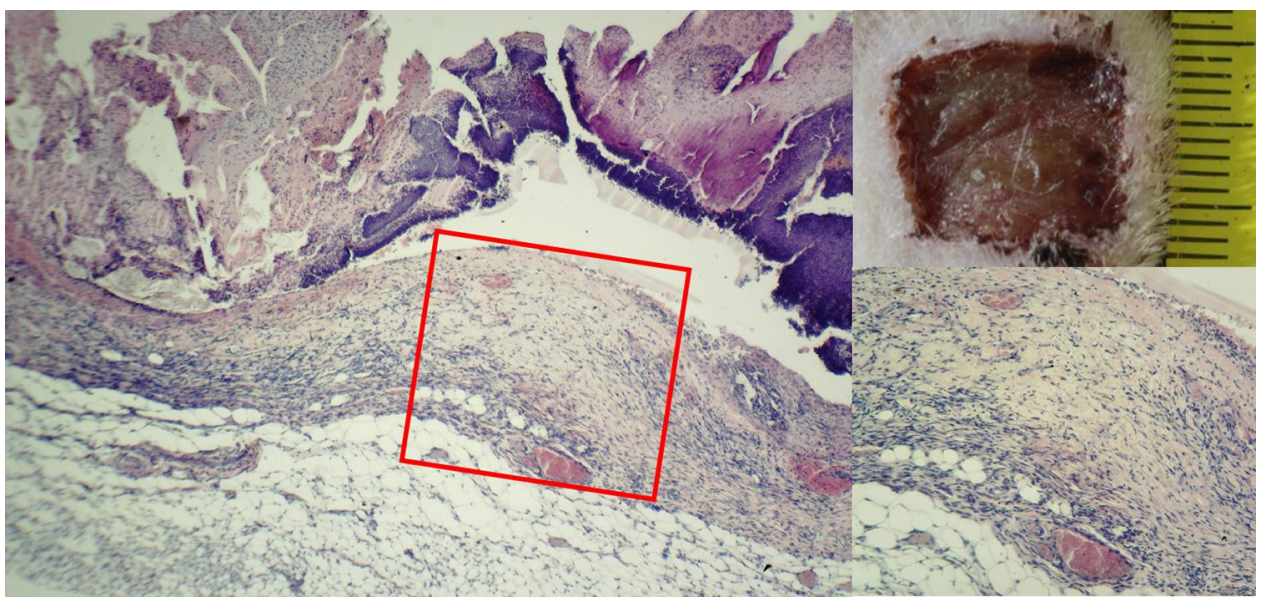

Figure 3 The wound crater center by the end of the 3rd day after wound modeling, Collagen group. Moderately present tissue edema and plethora, as well as moderate infiltration by leukocytes of all granulation layers. Hematoxylin-eosin staining. Magnification: X40 and XI00.

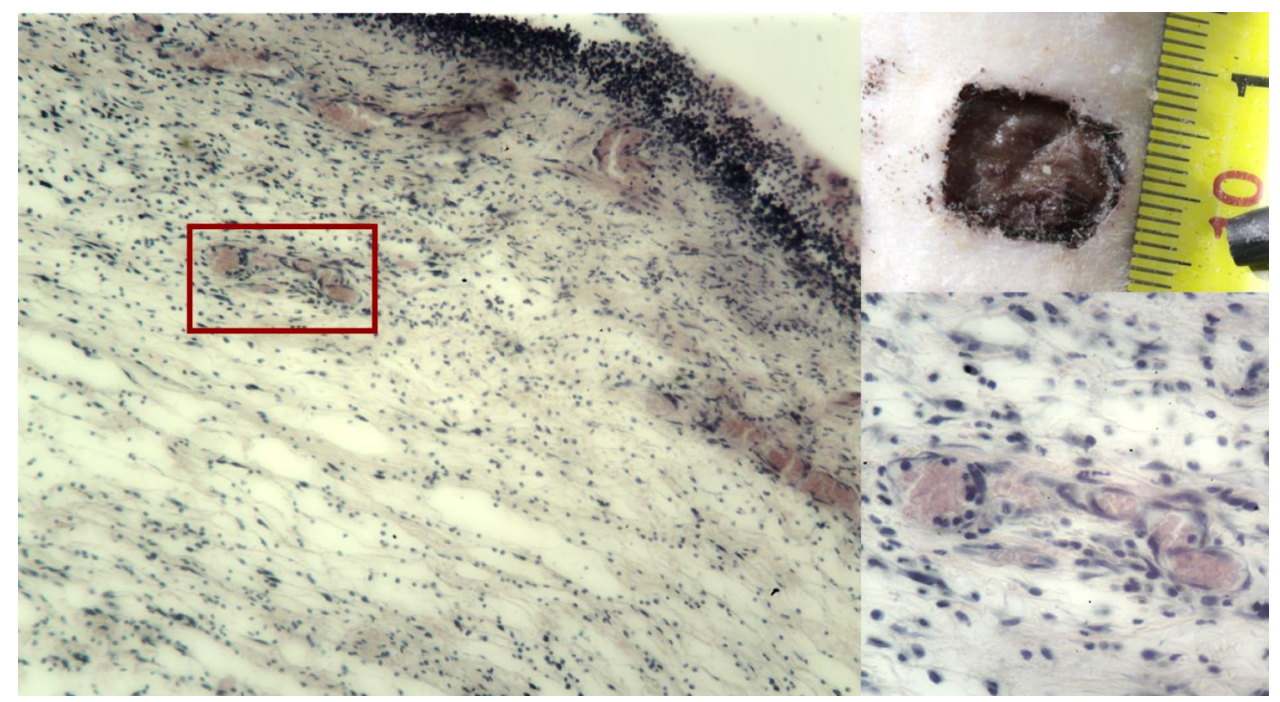

Figure 4 The wound crater center by the end of the 3rd day after wound modeling, SC group. The granulation tissue edema extends to deeper tissues. Hematoxylin-eosin staining. Magnification: $\times 40, \times 400$.

wound edge tissue on day 3, represented by $1-2$ layers of epithelial cells covering less than $10 \%$ of the wound surface.

\section{The Wound State by the End of the 7th Day of the Study}

The increase in the wound center temperature does not appear to be associated with an inflammatory process but instead with highly active regenerative processes.

Thus, the number of leukocytes in different granulation layers by the 7th day was minimal in the SC group, especially in the wound edges. In the wound edges, the SC group rats had the smallest absolute number of leukocytes $\left(\mathrm{Me}=186\right.$ cells per $\left.\mathrm{mm}^{2}\right)$. This number was statistically significantly less than that in the Control, Collagen, and DHB groups, which had approximately the same values for this indicator (Table 4).

It is important to highlight the differences in the leukocyte infiltration degrees of the wound edges and centers. In the wound centers of the SC group, the leukocyte numbers were 1.5 times higher than those in the periphery $(p<0.05)$, which confirms the anti-inflammatory effect of the SC culture. In the Collagen group, the leukocyte numbers in the wound center and edges were the same (on average, 308 and 302 cells $/ \mathrm{mm} 3$, respectively, $\mathrm{p}>0.05$ ). Conversely, in the DHB and Control groups, the leukocyte numbers were 1.1-1.2 times higher in the wound edges than in the center $(p<0.05)$.

In the wound tissue, the leukocyte numbers on the 7th day increased in all groups. At the same time, the 
Table 4 The Number of Leukocytes in the Center and Edges of Wounds On Day 7

\begin{tabular}{|l|l|l|}
\hline Groups & Center & Edges \\
\hline Control & $303[275: 307]$ & $349[325: 383]$ \\
Stem cells & $279[255: 292]$ & $186[176: 219] *$ \\
Collagen & $308[279: 350]$ & $302[266: 319] *$ \\
$\begin{array}{l}\text { Deproteinized hemoderivative } \\
\text { of calf blood }\end{array}$ & $266[233: 354]$ & $295[264: 359]$ \\
$\mathrm{P}$ & - & SC/Collagen \\
& & SC/GDB \\
\hline
\end{tabular}

Notes: Median [lower $25 \%$ quartile: upper $75 \%$ quartile].*- statistically significant difference from the control group (Mann-Whitney test, $\mathrm{p}<0.05$ ). P- significant difference between two unrelated treatment groups (Mann-Whitney test, $p<0.05$ ): SC/Collagen, Stem cells and Collagen; SC/DHG, Stem cells and Deproteinized hemoderivative of calf blood.

morphology of leukocytes changed, and neutrophilic infiltration was replaced by mononuclear cells and macrophages. The greatest increase (in comparison with the 3rd day) was observed in the concentration of the wound center leukocytes in animals with a high temperature level. The leukocyte numbers increased by 1.6 times in the SC group rats, 1.5 times in the Collagen group, 1.4 times in the Control group, and 1.3 times in the DHB group.

Thus, the influence of the drugs on wound center and edge leukocyte infiltration was observed only in animals of the SC group. In other groups (DHB and Collagen), there was no significant effect on the leukocyte response on day 7. Nevertheless, the animals treated with the pharmaceuticals demonstrated positive dynamics compared to the controls.

Microscopy of the wound center on the 7th day revealed tissue swelling, lymphatic vessel dilation, and full-blooded capillaries and venules. The severity of these processes within the groups decreased in the following order: SC, Collagen, DHB, and Control. Leukocyte infiltration was densest and most prevalent in the DHB group. In addition, on the 7th day after wound modeling, epidermal regeneration signs were observed in all wounds starting from the wound edges to the center. The least organized marginal shaft of the epidermis (according to the number of its layers) was found in the DHB group. In the SC group, the largest number of sites of future epidermal growth buds, from which hair, sebaceous glands, and other skin derivatives subsequently develop (Figure 5), were recorded.

By the 7th day the epidermis was not differentiated into layers in $57 \%$ of cases in the Control group, $43 \%$ of cases in the DHB and SC groups, and in $29 \%$ of cases in the Collagen group. Two layers of the epidermis were identified in $43 \%$ of cases in the Control group, $57 \%$ in the DHB and SC groups, and in $71 \%$ of cases in the Collagen group. Three layers of the epidermis on day 7 were not clearly visualized in all groups. The growth buds were identified in $14 \%$ of cases in the Control group, $43 \%$ of cases in the SK group and in $29 \%$ of cases in the Collagen group. In the DHB group growth buds were not detected.

\section{The Wound State by the End of the 14th Day of the Study}

The leukocyte numbers on the 14th day decreased compared to those on the 7th day by 1.2-1.9 times. By the end of the 2nd week, the leukocyte numbers were the lowest in the SC group, but the wound temperature in this group was the highest. In the wound center tissue on the 14th day, the number of leukocytes averaged 146 cells per $1 \mathrm{~mm}^{2}$ in the SC group (1.90 times less than that on the 7th day, $\mathrm{p}<0.01$ ); in the Control group, the leukocyte numbers decreased by 1.73 times compared to the numbers on the 7th day and averaged 172 non-resident cells per $\mathrm{mm}^{2}$ of wound bottom area on the 14th day, which was 1.18 times greater than that in the SC group $(p<0.05)$. In the Collagen group, the leukocyte numbers did not differ from the Control group on day 14. At the same time, they fell 1.64 times relative to day 7 and were 1.29 times higher than those in the SC group on the 14th day $(p<0.05)$. Wound center leukocyte infiltration was the most marked in the DHB group on the 14th day. This was the only group in which the leukocyte numbers did not change statistically compared to day 7. On average, the number of leukocytes in the DHB group was 224 per $\mathrm{mm}^{2}$ on the 14th day, which was 1.30 times greater than the amount in the Control group ( $\mathrm{p}<0.05$ ), 1.53 times more than that in the SC group $(\mathrm{p}<0.01)$, and 1.19 times more than that in the Collagen group $(\mathrm{p}<0.05)$. In the wound edge tissue, the number of leukocytes was the smallest in the SC group $(\mathrm{Me}=120$ leukocytes $/ \mathrm{mm} 2)$. SC was the only group in which the number of leukocytes on the 14th day in the wound edge tissue was significantly lower than that in the wound center tissue (1.21 times on average). In other groups, the number of leukocytes in the wound edges was comparable to those at the bottom of the wound (Table 5).

Thus, the injection of the stem cell culture into the wound tissue was accompanied by a decrease in leukocyte 

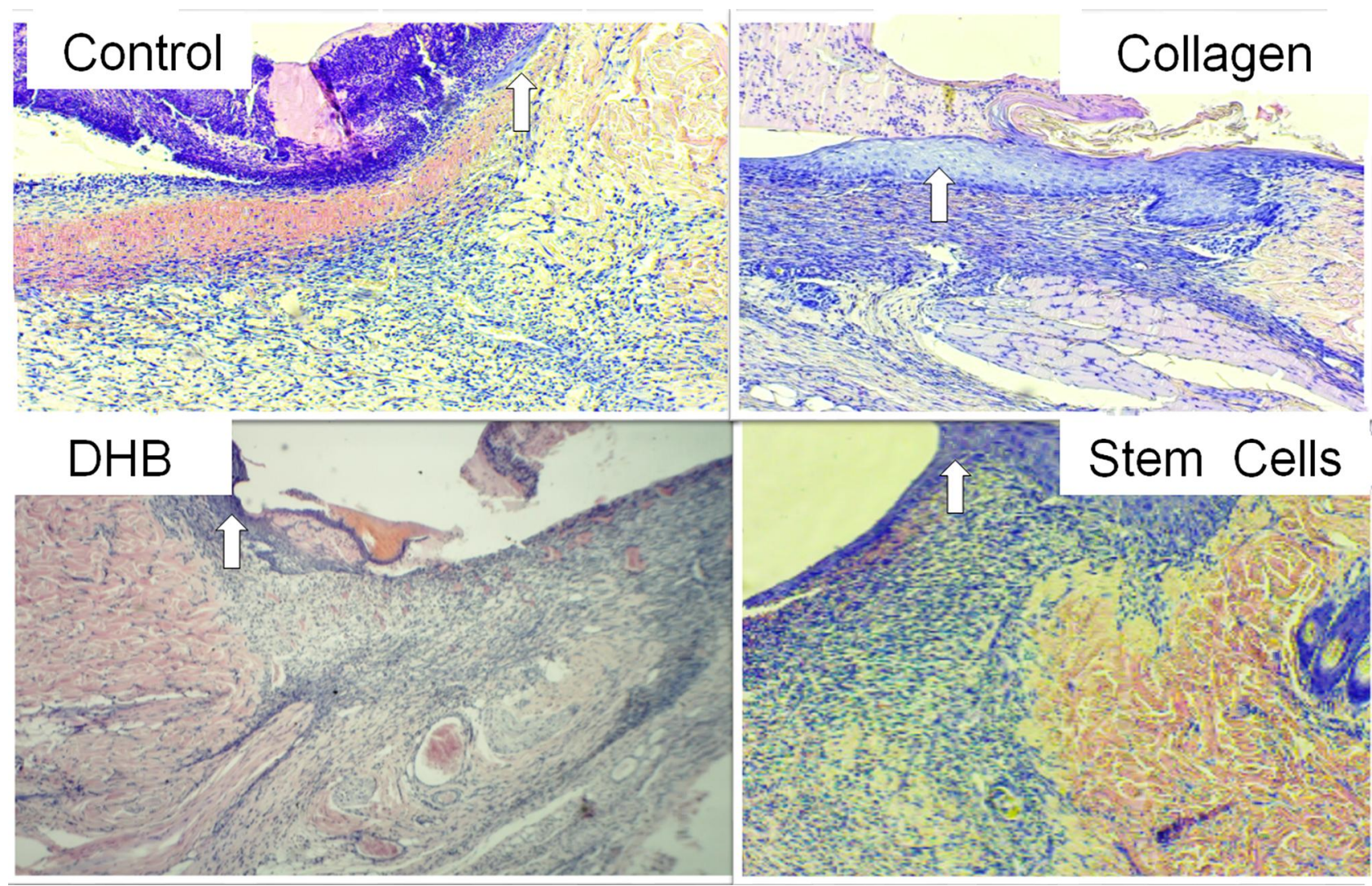

Figure 5 The wound edges in different groups on the 7th day of the study. Hematoxylin-eosin staining, magnification $x 100$. The arrows indicate the newly formed epithelium, which was wider and contained more layers in the SC and Collagen groups than that in the Control and DHB groups. In the SC and Collagen groups, the epithelium was multilayered, creeping from the edge to the center, and gradually becoming thinner. In the DHB and Control groups, there were signs of initial marginal epithelialization (a short section of a I-2 layered epithelium, which starts creeping from the wound edge).

infiltration for a long period of time (up to 14 days). The use of Collagen did not significantly affect the number of leukocytes in the wound bottom and edge tissue. DHB administration was characterized by an increase in leukocyte infiltration of the wound on the 14th day relative to the Control group.

An important morphological criterion for wound healing was the assessment of the epithelialization process by the end of the 2nd week. On the 14th day, epithelialization was marginal in all wounds of the Control and DHB groups. Complete wound coverage with epithelium was detected in $57 \%$ of cases in the SC group and in $43 \%$ cases in the Collagen group, which significantly distinguished those groups from the Control and DHB groups $(\mathrm{p}<0.05)$. In addition to weak wound epithelialization, the Control and DHB groups had pronounced scabs with leukocyte (mainly neutrophilic) infiltration. Under the newly formed multilayered epidermis, where the basement membrane is formed, the formation of growth buds of the sebaceous glands and hair follicles was observed. This is an important indicator of an optimal wound healing process. The most numerous and mature growth buds were observed in the SC and Collagen groups (Figure 6).

In all wounds, areas of the 3-layer epidermis were determined. But the smaller length of it was in the

Table 5 The Number of Leukocytes in the Center and Edges of Wounds on Day 14

\begin{tabular}{|l|l|l|}
\hline Groups & Center & Edges \\
\hline Control & $172[168: 195]$ & $188[142: 206]$ \\
Stem cells & $146[127: 164] *$ & $120[99: 142] *$ \\
Collagen & $188[166: 208]$ & $238[197: 242] *$ \\
Deproteinized & $224[207: 239] *$ & $196[162: 238]$ \\
hemoderivative of calf blood & & \\
$P$ & SC/Collagen & SC/Collagen \\
& SC/GDB & SC/GDB \\
& Collagen/GDB & \\
\hline
\end{tabular}

Notes: Median [lower $25 \%$ quartile: upper $75 \%$ quartile]. \# - the difference in the associated indicator dynamics within the same group compared to day 0 (Wilcoxon test, $\mathrm{p}<0.05$ ). *- statistically significant difference from the control group (MannWhitney test, $\mathrm{P}<0.05)$. $\mathrm{P}$ - significant difference between two unrelated treatment groups (Mann-Whitney test, $\mathrm{p}<0.05$ ): Collagen/DHG, Collagen and Deproteinized hemoderivative of calf blood; SC/Collagen, Stem cells and Collagen; SC/DHG, Stem cells and Deproteinized hemoderivative of calf blood. 


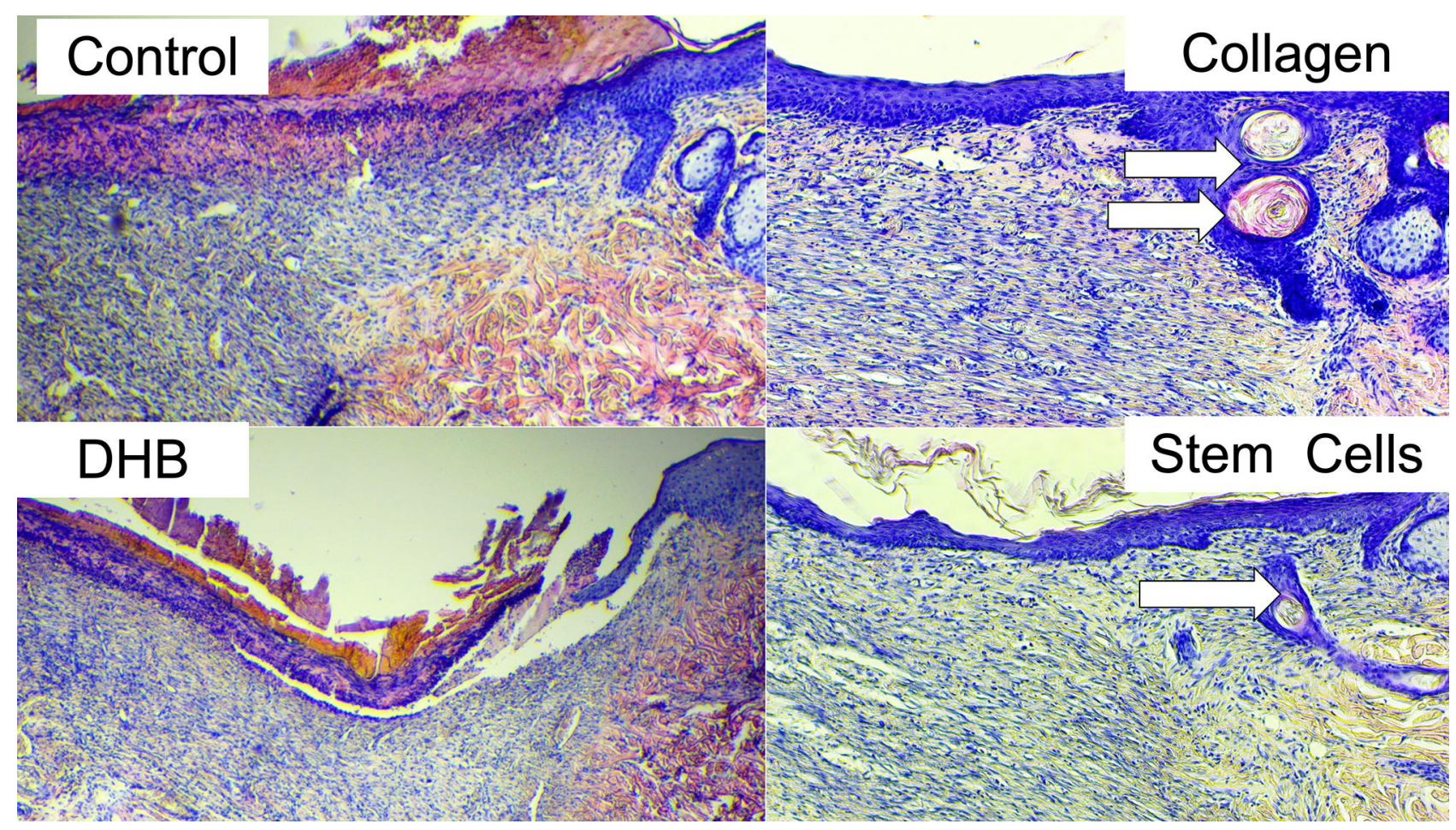

Figure 6 The wound edge view in different groups on the 14th day of the study. Arrows indicate the growth buds of the hair follicles. Hematoxylin-eosin staining, magnification $\times 100$.

Control and DHB groups. The growth buds and skin derivatives were identified in $100 \%$ of cases in the SC and Collagen groups, in $71 \%$ in the DHB group, and $57 \%$ in the Control group.

\section{Discussion}

The goal of this research was to determine the effect of inflammation on the acute skin wound healing process under the direct injection of stimulating agents via an experiment on rats.

It was necessary to determine whether a decrease in inflammation severity or an increase in inflammation duration (according to the degree of wound edge leukocyte infiltration, the time of onset and scab formation degree, and the wound surface epithelialization criteria) would accelerate the wound healing process when administering the agents via a solution containing stem cells, collagen, or DHB into the wound edges. To control additional trauma to the wound edges, isotonic $0.9 \%$ sodium chloride solution was introduced.

The observations show that at the end of the 3rd day after the same-size square skin wound modeling, the wound areas decreased only in the SC group. This decrease can be explained by the early transition from the exudation to the proliferation phase, which was confirmed by a decrease in wound tissue leukocyte infiltration. In the Collagen group, the wound area on the 3rd day remained the same as that on day 0 . However, leukocyte infiltration in the wound edge tissue was weaker than that in the Control group. By the end of the 3rd day, in the DHB and Control groups, the wound area was larger than that at the time of modeling. At the same time, in the control animals, leukocyte infiltration of the wound granulation tissue was the highest.

By the end of the 7th day, the wound areas decreased in all groups, except for the Control. The most prominent decrease was in the Collagen and SC groups, which histologically showed signs of a confident transition from the exudative inflammation phase to the proliferative one. This was accompanied by the lowest degree of wound tissue leukocyte infiltration, especially in the edges. The wound sizes in the Control group did not change compared to those on day 0 , and the histological signs of inflammation were the most pronounced in that group, as evidenced by the highest observable degree of leukocyte infiltration. At the same time, in the wound center, the leukocyte infiltration on the 7th day of the study was more marked than that on the 3rd day. However, morphologically, neutrophils were replaced by mononuclear cells and macrophages. 
The highest level of leukocyte infiltration with neutrophil predominance and the least pronounced wound epithelialization were recorded in the DHB group.

By the end of the 14th day of the study, the greatest signs of wound healing were observed in the Collagen and SC group rats. It was in these groups that the wound areas were the smallest and epithelialization was the most evident: The epithelium covered the entire surface of the former wounds in half of the animals. Simultaneously, we recorded the presence of a multilayer epithelium located on the formed basement membrane and the stratum corneum on the surface of the wounds, as well as the largest number of formed skin derivatives in the SC groups and in the Collagen group. In the DHB group, this effect was the same as that observed in the Control group. Complete wound epithelialization was not observed in any case. By the 14th day, scab discharge was observed two times more frequently in the SC and Collagen groups.

Another important fact is the consistently decreasing wound temperature during the first week of dynamic healing. This phenomenon has been linked to deceleration of the wound healing process. The wound center temperature continued to decrease by the 14th day in all groups, except for the SC. However, the weakest leukocyte infiltration was observed in the SC group, while at the edges of the wounds of the DHB group, the leukocyte numbers exceeded those in the Control group.

Thus, of all the drugs we used, an anti-inflammatory effect was only observed via the usage of mesenchymal stem cells, which agrees with the results of other authors. ${ }^{24-28}$ The use of the SC culture solution was accompanied by a decrease in the leukocyte infiltration degree up to 14 days, as well as a decrease in scabs and an acceleration in the transition from the exudation to proliferation phases. The above data suggest that the potentiation of the wound healing process (including inflammation as a component) in general and skin wounds in particular is largely the result of the factors synthesized by the injected mesenchymal stem cells, stimulating the proliferation and differentiation of a wide variety of cells (growth factors and other cytokines), including the area of inflammation. In the $\mathrm{SC}$ group, the leukocyte number was lower than that in the other groups, likely because of the high quantity of cytokines produced and stimulated by SC. Therefore, the need for leukocyte recruitment was reduced. Moreover, SCs and their metabolites are capable of inhibiting the excessive generation of reactive oxygen species and lipid peroxide reactions during inflammation. This ultimately had a positive effect on epithelialization and accelerated the wound healing rate.
The DHB solution usage in the acute skin wound modeling was characterized by a higher density of leukocyte infiltration into the wound center tissues on the 7th day, as well as an increase in the leukocyte numbers in the wound center tissues on the 14th day in comparison to all other experimental groups. This correlated with a decreased severity of wound epithelialization compared to the Collagen and SC groups. The relatively low-efficiency and pro-inflammatory effect of DHB could be a consequence of the introduction of the foreign proteins into the wound edges, subsequently spreading throughout the intercellular spaces, as well as inadequate changes in microcirculation. This possibly means that the absence of hypoxia led to a decreased effect of DHB. ${ }^{29,32}$

The use of collagen did not significantly affect the inflammation course or the wound tissue leukocyte infiltration levels. The acceleration of wound healing and epithelization may be associated with the stimulation of the proliferation phase and fibroblast functions (which would require a special study). The wound healing stimulated under collagen solution injections might result from the formation acceleration of the loose fibrous connective tissue matrix in the inflammation center. The introduction of exogenous collagen can improve wound healing via the application of a ready-made intercellular protein, albeit unstructured, which was effectively used by fibroblasts. Therefore, the effectiveness of collagen mechanisms will continue to be studied, especially since there is evidence of their positive effects under clinical use in surgery, cosmetology, and dentistry. ${ }^{34-39}$

\section{Conclusion}

Based on the results of this experimental study, the causal relationship between the inflammation and epithelialization processes during acute skin wound healing in rats was established.

The anti-inflammatory effect of SC injection into the wound edge tissue was determined, as well as the proinflammatory effect of DHB, with no effect on inflammation was observed under collagen treatment.

In comparison with the control group, a faster transition from the exudative to the proliferative phase of inflammation, as well as more intense wound epithelialization, were recorded in the SC and Collagen animal groups. Wounds of the DHB group healed worse.

\section{Abbreviations}

SC, stem cells; DHB, deproteinized hemoderivative of calf blood; Me, median. 


\section{Acknowledgments}

The authors thank Anikanov AV, Dudka VT, Furman YuV, Kaplin AN, Kryukov AA, and Naimzada M.D.Z. for their help and participation in the study.

The manuscript does not have a specific sponsorship. The materials presented were obtained as a part of the Foundation for the Promotion of the Development of Small Forms of Enterprises in the Scientific and Technical Field grant (232GRNTIS5/35963), devoted to the development of a biomedical product for wound healing. The groups described in the work were comparison groups for products developed and funded by the grant.

\section{Disclosure}

The authors report no conflicts of interest in this work.

\section{References}

1. Landén NX, Li D, Ståhle M. Transition from inflammation to proliferation: a critical step during wound healing. Cell Mol Life Sci. 2016;73(20):3861-3885. doi:10.1007/s00018-016-2268-0

2. Reinke JM, Sorg H. Wound repair and regeneration. Eur Surg Res. 2012;49(1):35-43. doi:10.1159/000339613

3. Sorg H, Tilkorn DJ, Hager S, Hauser J, Mirastschijski U. Skin Wound Healing: an Update on the Current Knowledge and Concepts. Eur Surg Res. 2017;58(1-2):81-94. doi:10.1159/000454919

4. Cañedo-Dorantes L, Cañedo-Ayala C-AM. Skin Acute Wound Healing: A Comprehensive Review. Int $J$ Inflammation. 2019;2019:3706315. doi:10.1155/2019/3706315

5. Serra MB, Barroso WA, da Silva NND, et al. From Inflammation to Current and Alternative Therapies Involved in Wound Healing. Int $J$ Inflammation. 2017;2017:3406215. doi:10.1155/2017/3406215

6. Eming SA, Martin P, Tomic-Canic M. Wound repair and regeneration: mechanisms, signaling, and translation. Sci Transl Med. 2014;6 (265):265sr6. doi:10.1126/scitranslmed.3009337

7. Wilgus TA, Roy S, McDaniel JC. Neutrophils and Wound Repair: positive Actions and Negative Reactions. Adv Wound Care. 2013;2 (7):379-388. doi:10.1089/wound.2012.0383

8. Vestweber D. How leukocytes cross the vascular endothelium. Nat Rev Immunol. 2015;15(11):692-704. doi:10.1038/nri3908

9. Dovi JV, Szpaderska AM, DiPietro LA. Neutrophil function in the healing wound: adding insult to injury? Thromb Haemost. 2004;92 (08):275-280. doi:10.1160/TH03-11-0720

10. Schafer M, Werner S. Oxidative stress in normal and impaired wound repair. Pharmacol Res. 2008;58(2):165-171. doi:10.1016/j. phrs.2008.06.004.

11. Lucas T, Waisman A, Ranjan R, et al. Differential roles of macrophages in diverse phases of skin repair. J Immunol. 2010;184 (7):3964-3977. doi:10.4049/jimmunol.0903356.

12. Hesketh M, Sahin KB, West ZE, Murray RZ. Macrophage Phenotypes Regulate Scar Formation and Chronic Wound Healing. Int J Mol Sci. 2017;18(7):1545. doi:10.3390/ijms18071545

13. Koh TJ, DiPietro LA. Inflammation and wound healing: the role of the macrophage. Expert Rev Mol Med. 2011;13:e23. doi:10.1017/ S1462399411001943.

14. Strbo N, Yin N, Stojadinovic SO. Innate and Adaptive Immune Responses in Wound Epithelialization. Adv Wound Care. 2014;3 (7):492-501. doi:10.1089/wound.2012.0435
15. Kim SY, Nair MG. Macrophages in wound healing: activation and plasticity. Immunol Cell Biol. 2019;97(3):258-267. doi:10.1111/ imcb.12236

16. Ridiandries A, Tan JTM, Bursill CA. The Role of Chemokines in Wound Healing. Int J Mol Sci. 2018;19(10):3217. doi:10.3390/ ijms19103217

17. Rodrigues M, Kosaric N, Bonham CA, Gurtner GC. Wound Healing: A Cellular Perspective. Physiol Rev. 2019;99(1):665-706. doi:10.1152/physrev.00067.2017

18. Mustoe TA, O??Shaughnessy K, Kloeters O. Chronic wound pathogenesis and current treatment strategies: a unifying hypothesis. Plast Reconstr Surg. 2006;117(SUPPLEMENT):35S-41S. doi:10.1097/01. prs.0000225431.63010.1b

19. Zhao R, Liang H, Clarke E, Jackson C, Xue M. Inflammation in Chronic Wounds. Int J Mol Sci. 2016;17(12):2085. doi:10.3390/ ijms 17122085

20. Xue M, Jackson CJ. Extracellular Matrix Reorganization During Wound Healing and Its Impact on Abnormal Scarring. Adv Wound Care. 2015;4(3):119-136. doi:10.1089/wound.2013.0485

21. Karppinen S-M, Heljasvaara R, Gullberg D, Tasanen K, Pihlajaniemi T. Toward understanding scarless skin wound healing and pathological scarring. F1000Res. 2019;8:F1000 Faculty Rev-787. doi:10.12688/f1000research.18293.1

22. Ogawa OR. Keloid and Hypertrophic Scars Are the Result of Chronic Inflammation in the Reticular Dermis. Int J Mol Sci. 2017;18(3):606. doi:10.3390/ijms 18030606

23. Sen CK, Gordillo GM, Roy S, et al. Human skin wounds: a major and snowballing threat to public health and the economy. Wound Repair Regen. 2009;17(6):763-771. doi:10.1111/j.1524-475X.20 09.00543.x

24. Su W-H, Wang C-J, Fu H-C, et al. Human Umbilical Cord Mesenchymal Stem Cells Extricate Bupivacaine-Impaired Skeletal Muscle Function via Mitigating Neutrophil-Mediated Acute Inflammation and Protecting against Fibrosis. Int $J$ Mol Sci. 2019;20(17):4312. doi:10.3390/ijms20174312

25. Wang L-T, Ting C-H, Yen M-L, et al. Human mesenchymal stem cells (MSCs) for treatment towards immune- and inflammationmediated diseases: review of current clinical trials. J Biomed Sci. 2016;23(1):76. doi:10.1186/s12929-016-0289-5

26. Li J, Li D, Liu X, Tang S, Wei F. Human umbilical cord mesenchymal stem cells reduce systemic inflammation and attenuate LPSinduced acute lung injury in rats. $J$ Inflamm. 2012;9(1):33. doi:10.1186/1476-9255-9-33

27. Prockop DJ, Oh JY. Mesenchymal stem/stromal cells (MSCs): role as guardians of inflammation. Mol Ther. 2012;20(1):14-20. doi:10.1038/mt.2011.211

28. Lee YS, Sah SK, Lee JH, Seo KW, Kang KS, Kim TY. Human umbilical cord blood-derived mesenchymal stem cells ameliorate psoriasis-like skin inflammation in mice. Biochem Biophys Rep. 2016;9:281-288. doi:10.1016/j.bbrep.2016.10.002

29. Guekht A, Skoog I, Edmundson S, Zakharov V, Korczyn AD. ARTEMIDA Trial (A Randomized Trial of Efficacy, 12 Months International Double-Blind Actovegin): A Randomized Controlled Trial to Assess the Efficacy of Actovegin in Poststroke Cognitive Impairment. Stroke. 2017;48(5):1262-1270. doi:10.1161/STROKEAHA.116.014321

30. Reichl F, Högg C, Liu F. Högg C., Liu F., et al. Actovegin ${ }^{\circledR}$ reduces PMA-induced inflammation on human cells. Eur J Appl Physiol. 2020;120(7):1671-1680. doi:10.1007/s00421-020-04398-2

31. Brock J, Golding D, Smith PM, Nokes L, Kwan A, Lee PYF. Update on the Role of Actovegin in Musculoskeletal Medicine: A Review of the Past 10 Years. Clin J Sport Med. 2020;30(1):83-90. doi:10.1097/ JSM.0000000000000566

32. Firan FC, Romila A, Onose OG. Current Synthesis and Systematic Review of Main Effects of Calf Blood Deproteinized Medicine (Actovegin $^{\circledR}$ ) in Ischemic Stroke. Int J Mol Sci. 2020;21(9):3181. doi:10.3390/ijms21093181 
33. Belikan P, Nauth L, Färber L-C, et al. Intramuscular Injection of Combined Calf Blood Compound (CFC) and Homeopathic Drug Tr14 Accelerates Muscle Regeneration In Vivo. Int $J$ Mol Sci. 2020;21(6):2112. doi:10.3390/ijms21062112

34. Dibirov MD, Gadzhimuradov RU, Koreyba KA. Analysis of the clinical application of epidermal growth factor ("Heberprot-P") and bioplastic material ("Collost") in treatment of skin and soft tissues defects in patients with diabetic foot syndrome. Khirurgiia. 2016;3:59-63. doi:10.17116/hirurgia2016359-63.

35. Sirak SV, Shchetinin EV, Sletov AA. Subantral augmentation with porous titanium in experiment and clinic. Stomatologiia. 2016;95 (1):55-58. doi:10.17116/stomat201695155-58

36. Silina EV, Stupin VA, Zolotareva LS, Komarov AN. Native collagen application in clinical practice for chronic wounds treatment. Khirurgiia. 2017;9:78-84. doi:10.17116/hirurgia2017978-84.
37. Stupin VA, Silina EV, Gorskij VA, et al. Efficacy and safety of collagen biomaterial local application in complex treatment of the diabetic foot syndrome (final results of the multicenter randomised study). Khirurgiia. 2018;6:1-100. doi:10.17116/hirurgia2018691-100

38. Manturova NE, Stenko AG, Petinati YA, Chaikovskaya EA, Bolgarina AA. Injectable collagen in correction of age-related skin changes: experimental and clinical parallels. Bulletin Russian State Med Univ. 2019;8(1):71-77. doi:10.24075/BRSMU.2019.010

39. Budkevich LI, Mirzoyan GV, Gabitov RB, Brazol MA, Salistyj PV. Collost bioplastic collagen material for the treatment of burns. Sovremennye Tehn Med. 2020;12(1):92-96. doi:10.17691/ stm2020.12.1.12

\section{Publish your work in this journal}

The Journal of Experimental Pharmacology is an international, peerreviewed, open access journal publishing original research, reports, reviews and commentaries on all areas of laboratory and experimental pharmacology. The manuscript management system is completely online and includes a very quick and fair peer-review system. Visit http://www.dovepress.com/testimonials.php to read real quotes from published authors. 